\title{
Disseminated Cutaneous Mycobacterium Fortuitum-Chelonae Complex Infection in a Young, Immunocompetent Patient: A Case Report
}

\author{
Atikah R. ${ }^{a}$, Kahairi A. ${ }^{a}$, Asha'ari, ZA ${ }^{\text {a }}$ \\ ${ }^{a}$ Department of Otorhinolaryngology, Head and Neck Surgery (ORL-HNS), Sultan Ahmad Shah Medical Centre (SASMEC) @IIUM
}

\author{
Keywords \\ Rapidly growing mycobacterium, \\ mycobacterium chelonae, mycobacterium \\ fortuitum, chronic skin infection, \\ immunocompetent \\ Corresponding Author \\ Assoc. Prof. Dr. Zamzil Amin \\ ORL-HNS Department, Level 5, \\ Kulliyyah of Medicine, International \\ Islamic University Malaysia, Bandar Indera \\ Mahkota Campus, Jalan Sultan Ahmad \\ Shah, 25200 Kuantan, Pahang Darul \\ Makmur \\ Tel No: +019-9564996 \\ E-mail: zamzilamin@iium.edu.my \\ Received: 2 March 2020; Accepted: 5 \\ March 2021 \\ Doi: https://doi.org/10.31436/imjm.v20i3
}

\begin{abstract}
Mycobacteria fortuitum and chelonae are a group of Rapidly Growing Mycobacteria (RGM) that can cause skin infections, most commonly in immunocompromised patients. RGM can also infect immunocompetent patients, but the disease is usually localized. Immunocompetent patients infected by RGM usually had a predisposing condition leading to the skin infection. We present a case of an immunocompetent patient with no predisposing factors, who presented with a chronic lesion on his neck that disseminated to his axilla. Culture and species identification from the skin biopsy revealed Mycobacterium fortuitum-chelonae complex. The patient was treated with a combination of surgery and multi-drug therapy. This case report highlights the rarity of cutaneous RGM infections encountered in ENT setting and the diagnostic dilemma due to the non-typical characteristics of skin lesion in RGM infections.
\end{abstract}

\section{INTRODUCTION}

Group IV of the Runyon classification for NTM consists of mycobacterium chelonae, fortuitum, and abscessus. They are known as rapidly growing mycobacteria (RGM) and although are ubiquitous in the environment, they are normally non-pathogenic. The type of cutaneous infection varies from patient to patient and depends on the patient's immune status. Due to the large number of differential diagnoses, this imposes a challenge to clinicians to achieve a correct diagnosis.

\section{CASE REPORT}

A 21-year old Malay gentleman presented with a painful ulcerative lesion on the right side of his neck which progressively increased in size within two years. It was initially neglected by the patient until it grew larger and ruptured, producing a foul-smelling discharge. There was no fever or constitutional symptoms. Past medical and surgical history was unremarkable. There was no family history of malignancy and no history of contact with Pulmonary Tuberculosis (PTB) patients. He denied high risk behaviours. There was no recent history of travelling or significant exposure to soil or aquatic environments.

Only when the lesion had become more extensive did the patient come for treatment. Examination showed a large mixed ulcerative-fungating lesion on the right side of his neck with involvement of the upper part of his right chest (Figure 1) and a foul-smelling serous discharge. The lesion was tender with some contact bleeding. Multiple bilateral cervical lymph nodes were palpable. Endoscopic examination was insignificant.

The patient was admitted for IV antibiotic therapy where he received IV amoxicillin-clavulanic acid $1.2 \mathrm{~g}$ three times daily. Blood tests showed mild leukocytosis and the erythrocyte sedimentation rate (ESR) was raised. Viral screening and autoimmune screening were negative. 


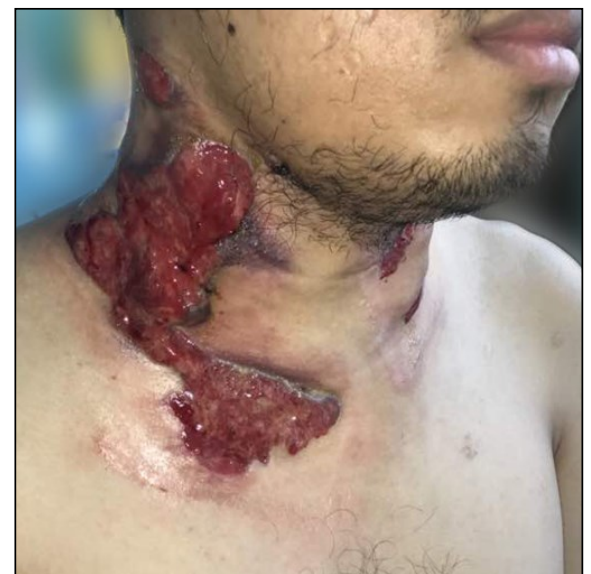

Figure 1: Large mixed ulcerative-fungating lesion of the neck

A contrasted computed tomography (CT) of the neck was done which showed multiple overlying skin breaks with a bulky right sternocleidomastoid and bilateral subcentimetre cervical lymph nodes but with no definite abscess formation (Figure 2). The non-specific findings represented either a chronic inflammation or infection. An incisional biopsy of the right neck lesion was performed. The histopathological examination (HPE) report came back as granulation tissue which was negative for Ziel-Niehlsen and Periodic Acid Schiff (PAS) stains.

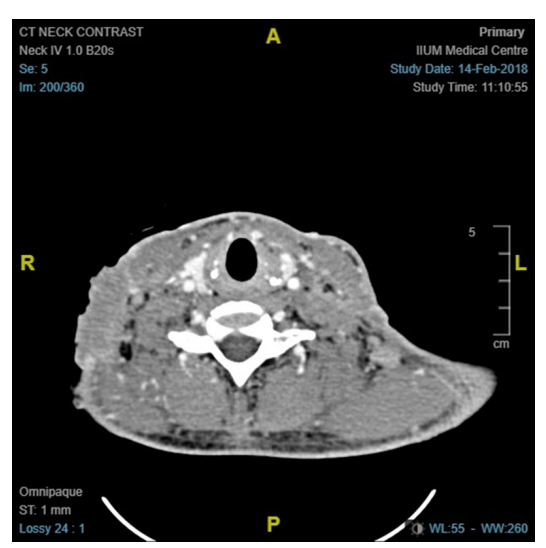

Figure 2: Contrasted CT neck showing multiple overlying skin breaks with a bulky right sternocleidomastoid muscle.

Following the incisional biopsy, the patient had repeated admissions to the hospital for debridement. The lesion had extended to involve the left side of his neck. It also extended caudally and developed into a right axillary abscess which required drainage. Specimens from the drainage all yielded negative results (tissue, pus C\&S, and acid-fast bacilli).
The result for MTB C\&S from the neck lesion was reported and showed positivity for non-tuberculous mycobacterium. Species identification via conventional culture method revealed Mycobacterium FortuitumChelonae complex. A chest physician with vast experience in managing NTM infections was consulted for input regarding treatment options. The patient was started on oral ciprofloxacin 500mg twice daily and oral azithromycin $500 \mathrm{mg}$ once daily, both for the course of 6 months in which he responded well to. After completing 6 months of treatment, the patient had completely recovered.

\section{DISCUSSION}

Non-tuberculous mycobacteria (NTM) is divided into four groups based on the Runyon system. Groups I through III are slow growing. The Runyon IV organisms, also known as rapidly growing mycobacteria (RGM), include mycobacterium chelonae, fortuitum, and abscessus. RGM are ubiquitous in the environment and can be found in soil and natural water supplies. They commonly are not pathogenic, but can infect a person through puncture wounds.

RGM can cause infections following invasive procedures such as subcutaneous injections of traditional medicine, excision of a sebaceous cyst, Ibrutinib therapy, skin graft, cosmetic surgery, and sclerotherapy of a varicose vein. 1,2,3,4,5 Health-care associated outbreaks is believed to be due to contaminated tap water. Environmental exposure such as gardening has also been shown to cause cutaneous RGM infections. What is unique about our case is that our patient is a young, immunocompetent patient who had no previous exposure to the risks mentioned above.

In young individuals, the most common aetiology of cutaneous infections caused by RGM is M. fortuitum. In older individuals, M. chelonae and M. abscessus are more commonly isolated. Cutaneous infection by RGM has a wide variety of clinical presentations, ranging from asymptomatic to tender erythematous nodules, cellulitis, ulcers, and draining sinuses. ${ }^{7}$ In an immunocompetent person, symptoms are usually localized. In our patient however, although immunocompetent, his disease had 
disseminated to the axilla due to treatment delay. Had he not delayed his presentation to the hospital, the disease could have been treated earlier and might have been contained in the neck area. Disseminated and severe lesions commonly occur in those who are immunocompromised or immunosuppressed. 8 ,9

The vast range of symptoms imposes a challenge for clinicians as they can create a diagnostic dilemma. This may cause a delay at initiating treatment hence allowing time for the infection to disseminate. In our patient, the unfamiliarity with the nature of RGM skin infections caused us not to have suspicion of RGM as the causative agent. We initially suspected squamous cell carcinoma due to the ulcerative feature of the skin lesion. To confirm the diagnosis of cutaneous RGM infections, multiple biopsies of the skin lesion and cultures are essential. Histologically, the lesions are characterized by granulomatous reactions. In cases with abscess formation, neutrophil predominance may be observed.1,5,9 The sensitivity of AFB staining is unsatisfactory in majority of RGM cases, although it is a fast and convenient diagnostic method. Therefore, despite being time-consuming, cultures are needed for mycobacteria detection, which also allows susceptibility testing.

Treatment for a cutaneous RGM infection is not standardized, but a combination of surgical treatment with antimicrobial therapy is recommended. All abscesses should be drained and unhealthy tissue debrided. Our patient had undergone surgical debridement multiple times to provide a healthy wound bed for healing process to take place. The type and duration of antimicrobial therapy depend on the severity of infection and immune status of the patient. For the time being there are no statistically significant clinical trials that compare the efficacies of RGM-specific antibiotics, thus current guidelines suggest for susceptibility testing. Jayasingam et al have studied the pattern of resistance of antibiotics commonly prescribed for RGM infections (amikacin, ciprofloxacin, clarithromycin, imipenem, linezolid), but the RGM isolated were from the lower respiratory tract and not from skin biopsies. ${ }^{10}$ Current literature on treatment of cutaneous RGM infections supports the use of multidrug therapy to avoid resistance and a long-term therapy to prevent recurrences.
The flaw in our management was that following mycobacterium species identification, treatment was initiated without antimicrobial susceptibility testing. The choice of combination of azithromycin and ciprofloxacin was not based on drug sensitivity, but was started after consultation with a chest physician. Fortunately, the patient responded well to the treatment. It is recommended however, that susceptibility testing be performed in all future cases of RGM infections so that a more targeted therapy can be given.

We report this case due to the rarity of such reported cases in our population and the peculiarity of the host in which the patient is a young, immunocompetent patient with no identifiable risk factors, as well as the diagnostic dilemma due to the wide range of symptoms in RGM infections. The clinical presentation of the patient was unique as he presented with a disseminated skin infection which is not commonly seen in immunocompetent patients.

\section{REFERENCES}

1. Tsai S, Chen L, Liao H, Chiang C, Lin W. Case Report: Complicated skin and soft tissue infection with Mycobacterium fortuitum following excision of a sebaceous cyst in Taiwan. J Infect Dev Ctries. 2016;10(12):1357-61.

2. Dousa KM, Babiker A, Aartsen D Van, Shah N. Ibrutinib therapy and Mycobacterium chelonae skin and soft tissue infection. OFID. 2018;10 (1093):1-3.

3. Smith BD, Liras IN, Cicco IA De, Aisenberg GM. Mycobacterium fortuitum infection of the scalp after a skin graft. BMJ. 2016; doi:10.1136/bcr-2016 $-216968$

4. Maurer FP, Castelberg C, Braun Von A, Wolfensberger A, Bloemberg G V., Böttger EC, et al. Postsurgical wound infections due to rapidly growing mycobacteria in Swiss medical tourists following cosmetic surgery in Latin America between 2012 and 2014. Eurosurveillance. 2014;19 (37):1-4.

5. Filho GH, Takita LC. Disseminated cutaneous atypical mycobacteriosis by M. chelonae after sclerotherapy of varicose veins in an 
immunocompetent patient: A case report. An Bras Dermatol. 2015;3(90):138-42.

6. Uslu U, Böhm O, Heppt F, Sticherling M. Skin and soft tissue infections caused by Mycobacterium chelonae : Acta Derm Venereol. 2019;99:889-93.

7. Zhang L, Liu W, Jiang H, Zhong W, Zhang G, Jin P WH. Cutaneous Infections Caused by Rapidly Growing Mycobacteria: Case Reports and Review of Clinical and Laboratory Aspects. Acta Derm Venereol. 2015;95:985-9.

8. Ana MP, Leonor G. Mycobacterium chelonae Is an Ubiquitous Atypical Mycobacterium. Case Rep Dermatol. 2015;7:207-11.

9. Dubow A, Morand M, Désy D, Krasny M. Recurrence of cutaneous Mycobacterium chelonae infection: A case report. JCMS Case Reports. 2019;7:1-3.

10. Jayasingam SD, Zin T, Ngeow YF. Antibiotic Resistance in Mycobacterium Abscessus and Mycobacterium Fortuitum Isolates from Malaysian Patients. Int J Mycobacteriology. 2017;6 (4):387-90. 